(c) 2019 Universidad Nacional Autónoma de México, Facultad de Estudios Superiores Zaragoza.

Este es un artículo Open Access bajo la licencia CC BY-NC-ND (http://creativecommons.org/licenses/by-nc-nd/4.0/).

TIP Revista Especializada en Ciencias Químico-Biológicas, 22: 1-9, 2019.

DOI: $10.22201 /$ fesz.23958723e.2019.0.180

\title{
Aspectos generales del transporte de colesterol en la esteroidogénesis de la placenta humana
}

\author{
Sofía Olvera-Sánchez, Mercedes Esparza-Perusquía, \\ Oscar Flores-Herrera, Viviana A. Urban-Sosa y Federico Martínez* \\ Departamento de Bioquímica, Facultad de Medicina, Universidad Nacional Autónoma de México, \\ Alcaldía de Coyoacán, 04510, Ciudad de México, México. E-mail: *fedem@bq.unam.mx
}

\begin{abstract}
RESUMEN
La placenta humana requiere de colesterol para sintetizar la progesterona que mantiene la relación entre el feto y la madre, lo que le permite concluir de manera exitosa el embarazo. La placenta incorpora el colesterol principalmente a través de las lipoproteínas de baja densidad (LDL) que se obtienen del torrente circulatorio materno por un mecanismo de endocitosis. A los endosomas que se generan en este proceso se les unen varias proteínas conformando los endosomas tardíos, que degradan las LDL y liberan el colesterol a las mitocondrias del sinciciotrofoblasto que lo transforman en pregnenolona y posteriormente en progesterona. Las proteínas de fusión de membranas denominados complejos SNARE participan en la liberación del colesterol en sitios de contacto específicos en donde se localizan las proteínas mitocondriales responsables de la esteroidogénesis.

Palabras Clave: placenta humana, receptores de lipoproteínas, mitocondrias, transporte de colesterol, esteroidogénesis, sitios de contacto.
\end{abstract}

\section{General aspects of cholesterol transport in the steroidogenesis of the human placenta}

\begin{abstract}
The human placenta requires cholesterol to synthesize the progesterone that maintains the relationship between the fetus and the mother, which allows it to successfully conclude pregnancy. The placenta incorporates cholesterol through the LDL obtained from the maternal blood stream by a mechanism of endocytosis. Endosomes formed by this process degrade the LDL, bind several proteins forming the late endosomes and releasing the cholesterol to syncytiotrophoblast mitochondria to transform it into pregnenolone and then, into progesterone. The soluble attachment proteins denominated SNARE participates in the transport of cholesterol in specific contact sites where the mitochondrial proteins responsible for steroidogenesis are located.
\end{abstract}

Key Words: human placenta, lipoprotein receptors, mitochondria, cholesterol transport, steroidogenesis, contact sites.

Nota: Artículo recibido el 08 de diciembre del 2018 y aceptado el 17 de junio del 2019. 


\section{INTRODUCCIÓN}

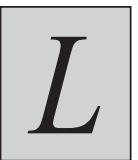

a placenta humana tiene un papel primordial al establecer mecanismos de comunicación entre el feto y la madre durante el embarazo. En este proceso, la progesterona adquiere un papel central al favorecer la implantación del blastocisto, la remodelación de la matriz extracelular y la promoción de la migración del trofoblasto (Halasz \& Szekeres-Bartho, 2013). Además, la síntesis de progesterona se incrementa conforme avanza el embarazo (Morel et al., 2016). Estas funciones garantizan que el producto alcance la madurez que le permita vivir fuera del vientre materno (Burton \& Fowden, 2015; Vaughan \& Fowden, 2016; Ashary, Tiwari \& Modi, 2018).

La placenta es un órgano excepcional capaz de realizar de manera simultánea las funciones de diferentes tejidos, por ejemplo: sintetiza hormonas como las glándulas endocrinas, realiza el intercambio gaseoso como en los pulmones y filtra metabolitos como en los riñones, entre otras funciones (Chatuphonprasert, Jarukamjorn \& Ellinger, 2018). A pesar del papel que desempeña la placenta, hasta el momento no se conocen con certeza los mecanismos maternos o fetales que la controlan, aunque se han sugerido posibles señales autocrinas, paracrinas o intracrinas. El linaje celular funcional de la placenta es el trofoblasto, que se divide en cito y sinciciotrofoblasto, este último es responsable del transporte de nutrientes y desechos al estar en contacto con las lagunas maternas y al mismo tiempo, el responsable del metabolismo esteroidogénico (en este artículo se considera la esteroidogénesis como la transformación del colesterol en progesterona). Si bien no se conocen varios aspectos del control del metabolismo placentario, lo que sí es claro es que la placenta humana tiene un papel central en las funciones del embarazo (Byrns, 2014), para mantener la relación maternofetal al sintetizar cantidades elevadas de progesterona, una hormona esteroide que se produce en las mitocondrias del sinciciotrofoblasto y que regula, entre otras funciones, los procesos inmunológicos que evitan que el producto sea rechazado, ya que la carencia o disminución de progesterona podría provocar un aborto (Holland et al., 2017a).

Se ha demostrado que los explantes de la placenta humana (pequeños fragmentos que contienen a las células del trofoblasto), son resistentes a condiciones físicas no fisiológicas que un tejido "normal" no podría soportar. Por ejemplo, luego de la incubación en un medio hipotónico o a temperaturas superiores a $\operatorname{los} 45^{\circ} \mathrm{C}$, los explantes realizan todas sus funciones metabólicas, incluyendo la síntesis de progesterona, de manera similar a los explantes que no fueron expuestos a estos tratamientos. Los resultados muestran que la placenta humana contiene mecanismos de autopreservación y como estrategia fisiológica, un control metabólico y funcional independiente a los estímulos externos que desarrolló como una forma para proteger al producto (Paul, Jailkhani \&Talwar, 1978, Paul, Gupta, Jailkhani \&Talwar, 1980). En este sentido, se ha reportado que las mitocondrias de la placenta se adaptan de acuerdo con el avance del embarazo (Holland et al., 2017b). Por otro lado, aunque existen abundantes evidencias del metabolismo energético, aún falta mucho por conocer del funcionamiento de la placenta en el ser humano (Kaiser, 2014; Bianco-Miotto et al., 2016).

\section{RECEPTORES A LIPOPROTEÍNAS}

Para la síntesis de progesterona es necesario el colesterol; sin embargo, debido a que la placenta no tiene la maquinaria para sintetizar las cantidades de colesterol que se requieren para la producción diaria de progesterona (van Leusden \& Villee, 1965; Zelewski \& Villee 1966), éste es proporcionado por las lipoproteínas maternas a través de los receptores específicos del sinciciotrofoblasto. En las células del trofoblasto se ha descrito la presencia de varios tipos de receptores para las lipoproteínas, como el de las lipoproteínas de baja densidad (LDL) que son los que se expresan de manera principal (Winkel, Snyder, MacDonald \& Simpson,1980; Furuhashi et al., 1989; Chatuphonprasert et al., 2018). Las lipoproteínas de alta densidad (HDL) se han identificado en las células del trofoblasto (Wadsack et al., 2003), en las células BeWo (Pagler et al., 2006) y en varios tejidos esteroidogénicos. Sin embargo, a diferencia de las LDL, las HDL se procesan por una vía diferente a la lisosomal (Sanderson, 2009).

Debido a que la síntesis de progesterona está sujeta a la capacidad de incorporar el colesterol de las LDL (Figura 1), se determinó que la concentración de los receptores para las lipoproteínas está regulada por la presencia de las mismas LDL. En células aisladas del trofoblasto y mantenidas en cultivo, la incubación con LDL-I ${ }^{125}$ por tiempos largos, disminuyó cerca del 90\% la captación y degradación de las LDL, lo que sugiere que las células del trofoblasto pueden regular el número de receptores de las LDL en la membrana plasmática. Este efecto no se observó cuando las células se incubaron en presencia de HDL (Winkel, MacDonald \& Simpson, 1981). Sin embargo, datos recientes sugieren que la placenta usa tanto las LDL como las HDL para obtener el colesterol necesario para la síntesis de progesterona (Sanderson, 2009). La capacidad de la placenta de emplear ambas lipoproteínas asegura que el aporte de colesterol sea constante para mantener la esteroidogénesis, aun en condiciones que puedan afectar la concentración de cualquiera de éstas, asegurando así que el embarazo no esté en riesgo.

El aporte del colesterol materno no se usa tan solo para la producción de progesterona, ya que también se requiere para satisfacer las necesidades del desarrollo del feto en crecimiento (Woollett, 2011; Bartels \& O'Donoghue, 2011; Bhattacharjee et al., 2012; Baardman et al., 2013). 


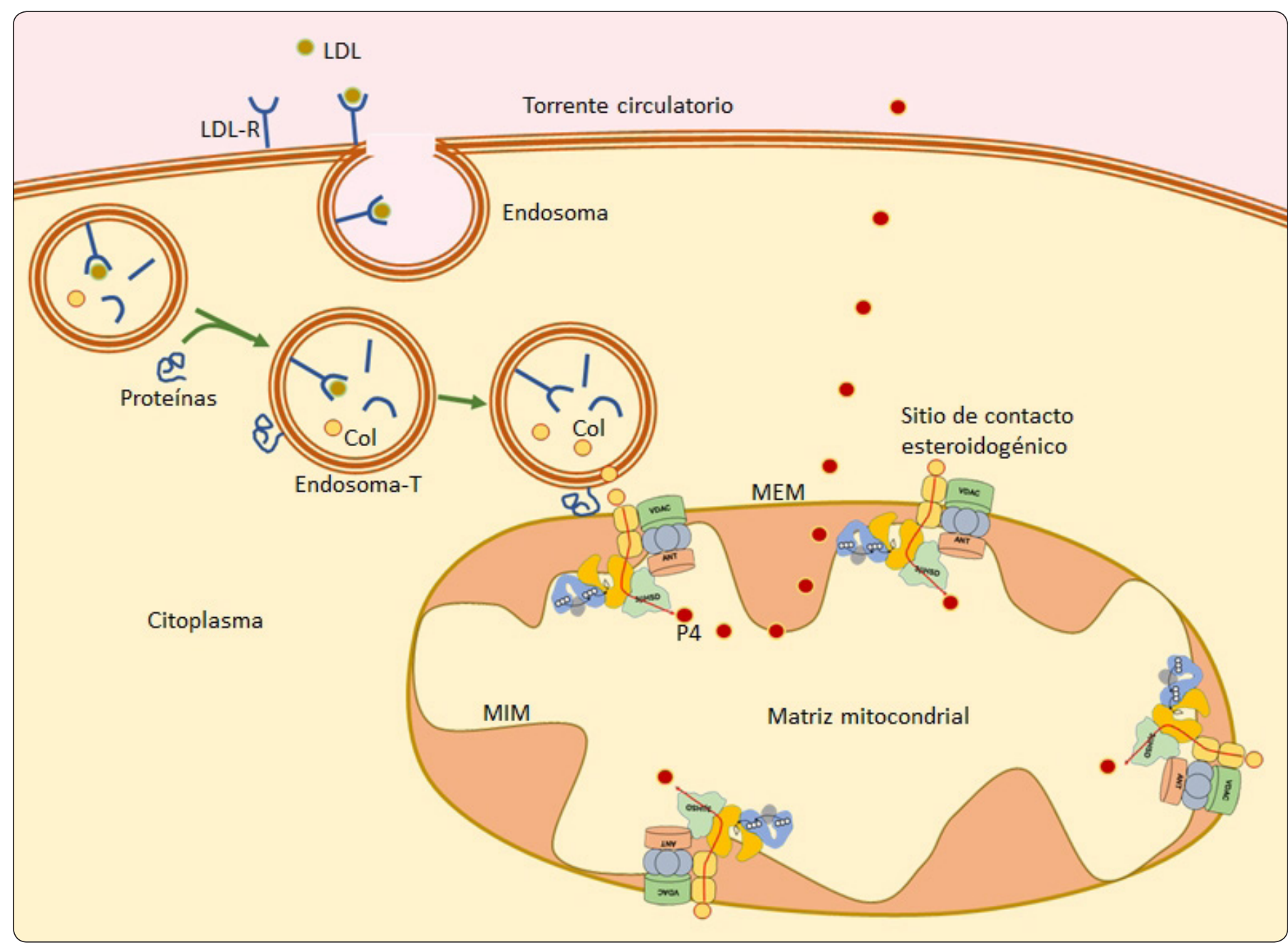

Figura 1. Esquema de transporte del colesterol en el torrente circulatorio materno a las mitocondrias de las células de la placenta humana. Las LDL maternas se asocian a los receptores de las LDL (LDL-R) que son endocitadas conformando el endosoma, en donde el colesterol se libera de las LDL. Posteriormente, se asocian proteínas para constituir el endosoma tardío (Endosoma-T) que permite su asociación a la membrana externa mitocondrial (MEM), en donde se encuentran los sitios de contacto esteroidogénicos. En estos sitios se realiza el transporte de colesterol que ingresa a la membrana interna en donde se localiza la cadena del citocromo P450sce para su transformación en pregnenolona (P5) y posteriormente a progesterona (P4). Una vez sintetizada la $\mathbf{P 4}$, ésta se libera al torrente circulatorio para mantener la relación materno-fetal. Figura elaborada por los autores.

\section{TEJIDOS ESTEROIDOGÉNICOS Y TRANSPORTE MITOCONDRIAL DEL COLESTEROL}

Aunque el colesterol es una molécula hidrofóbica que las células requieren para varias funciones, su concentración es altamente regulada, ya que los cambios en las concentraciones pueden ser fatales para la viabilidad celular, como en el caso de la estabilidad de las membranas. Se ha descrito que, en las células de los mamíferos, la presencia de colesterol en el retículo endoplásmico es baja, pues su concentración corresponde al 5\% de los fosfolípidos; sin embargo, en el aparato de Golgi su concentración es mayor al 30\% (Mesmin, Antonny \& Drin, 2013). La mayoría del colesterol se localiza en la membrana plasmática, con un $60 \%$ del total del esterol celular. Las diferencias en la concentración del colesterol en las membranas sugieren la existencia de un mecanismo de regulación celular mediado por al menos dos vías de transporte: vesículas y proteínas específicas (Flis \& Daum, 2013).

En los tejidos esteroidogénicos, el transporte de colesterol al citocromo P450scc mitocondrial es relevante, ya que cuando se estimula la síntesis de pregnenolona, la primera hormona esteroide que se genera por la actividad de la cadena acoplada al P450scc, el transporte de colesterol hacia la mitocondria deber ser vectorial, eficiente y en la concentración necesaria para dar respuesta a las necesidades celulares y sin alterar sus funciones (Chien, Rosal \& Chung, 2017). 
Dentro de las vías de transporte del colesterol celular, se ha descrito una familia de proteínas denominadas STARD, con capacidad de unir al colesterol y participar en su distribución intracelular (Alpy et al., 2013). Estas proteínas se asocian a complejos multiproteicos específicos que aseguran la precisa distribución del esterol. Aunado a esto, se han descrito asociaciones entre las membranas de los diferentes organelos celulares (ver más adelante), conformados por transportadores, enzimas y proteínas de andamiaje (Mesmin, Antonny \& Drin, 2013), formando microdominios entre las membranas denominados sitios de contacto (Poderoso et al., 2013; Soffientini \& Graham, 2016).

Se han descrito varios tipos de sitios de contacto, por ejemplo, las membranas asociadas a las mitocondrias (mitochondriaassociated ER membrane, MAMs), que son un microdominio de asociación entre el retículo endoplásmico (RE) y las mitocondrias. Es importante destacar que la mitocondria tiene un papel relevante en el proceso esteroidogénico, ya que es ahí en donde reside la transformación del colesterol en pregnenolona (Papadopoulos \& Miller, 2012), además de participar en otros procesos (Tait \& Green, 2012), como la síntesis de ATP, señalización intracrina, la apoptosis, la inmunidad innata, la autofagia o la respuesta al estrés (Galluzzi, Kepp \& Kroemer, 2012). Este tipo de microdominios se ha sugerido que contribuye al transporte de diferentes lípidos entre las membranas celulares.

En la esteroidogénesis, parecen conjuntarse un sistema mediado por vesículas y otro por proteínas. Dentro del sistema proteico, se ha descrito a la STARD1 como la principal proteína que participa en la esteroidogénesis de gónadas y glándulas suprarrenales. Se ha demostrado que la STARD1 transfiere colesterol eficientemente a la mitocondria (Tuckey, Headlam, Bose \& Miller, 2002) y que su interacción con el colesterol induce un cambio conformacional de su estructura nativa a glóbulo fundido ('molten globule'), que se ha sugerido como un estado dinámico que permite la transferencia del esterol (Rajapaksha, Kaur, Bose, Whittal \& Bose, 2013). En la interacción de la STARD1 con la mitocondria durante el transporte del colesterol se han reportado complejos multiproteicos formados por un grupo de diversas proteínas entre las que se encuentran la VDAC (Bose, Whittal, Miller \& Bose, 2008; Prasad et al., 2015), el receptor $\sigma-1$ (Marriott, Prasad, Thapliyal \& Bose, 2012) y la ATPasa ATAD3 (Issop et al., 2015), por mencionar algunas. En este sentido, los complejos multiproteicos a su vez pueden interaccionar con elementos proteicos del retículo endoplásmico, con gotas lipídicas o endosomas tardíos (Beller, Thiel, Thul \& Jäckle, 2010; Yang, Galea, Sytnyk \& Crossley, 2012).

Aunque hay un gran flujo de colesterol en los tejidos esteroidogénicos de regulación aguda, en las mitocondrias la cantidad de colesterol es baja (Horvath \& Daum, 2013); sin embargo, en las mitocondrias de la placenta humana los niveles de colesterol son altos, semejantes incluso a los de la membrana plasmática (Navarrete, Flores-Herrera, Uribe \& Martínez, 1999). Esto adquiere relevancia, ya que hasta el momento no se han descrito moduladores de la esteroidogénesis placentaria; sin embargo, esta característica habla de un transporte permanente de colesterol que permite mantener una producción de progesterona de manera sostenida durante el embarazo.

Una vez que el colesterol ha sido transportado a la membrana externa mitocondrial, éste debe continuar su tránsito hasta la membrana interna donde se encuentra el citocromo P450scc para ser transformado en pregnenolona (Martínez $\&$ Strauss, 1997). Esto sugiere que un paso limitante en la esteroidogénesis podría ser la transferencia de colesterol de la membrana externa a la interna mitocondrial. Se ha reportado que para el transporte de colesterol entre las membranas mitocondriales se requiere de los sitios de contacto (Strauss, Kishida, Christenson, Fujimoto \& Hiroi, 2003; Miller \& Bose, 2011).

No obstante que la STARD1 es la principal proteína asociada al flujo de colesterol hacia la mitocondria y descrita para diferentes tejidos esteroidogénicos de regulación aguda, la placenta humana no la expresa (Miller, 2013). Sin embargo, la placenta sintetiza entre 250 y $500 \mathrm{mg}$ de progesterona al día durante el último trimestre del embarazo (Simpson \& MacDonald, 1981; LaVoie \& King, 2009); esto implica un flujo continuo de colesterol hacia la mitocondria. En la última década se identificó en las células del sinciciotrofoblasto a la proteína STARD3 (Watari et al., 1997), descrita originalmente en un tumor de mama, la cual presenta el dominio START de unión al colesterol característico de esta familia de proteínas.

La STARD3 es una proteína endosomal con 4 segmentos transmembranales en su extremo amino terminal (dominio MENTAL). Alpy \&Tomasetto (2014), reportaron que, aunque la STARD1 y STARD3 presentan un dominio START y comparten ciertas propiedades bioquímicas, también son diferentes en varios aspectos, que van desde su localización hasta su estructura (van der Kant, Zondervan, Janssen \& Neefjes, 2013) demostraron que los endosomas tardíos y los lisosomas contienen la mitad del colesterol celular y que de ellos depende su distribución. También observaron que hay dos tipos de endosomas tardíos, uno presenta a la proteína ORP1L que transporta oxisteroles y otro contiene a la STARD3 y a la ABCA3 (una proteína transportadora que usa ATP para la translocación de sustratos, incluyendo lípidos), (van der Kant, Zondervan, Janssen \& Neefjes, 2013). Esto les permitió sugerir que la STARD3 facilita el flujo de colesterol de los endosomas tardíos a la mitocondria, constituyendo un tipo de transporte vesicular. De igual manera, se ha descrito que la STARD3 requiere de los aminoácidos M307 y N311 
para unir al colesterol (van der Kant, Zondervan, Janssen \& Neefjes, 2013). Asimismo, construyeron el knock-out del gen que codifica para la STARD3 y observaron que no se eliminó de manera total la síntesis de progesterona, sugiriendo que el transporte de colesterol mediado por la STARD3 no es el único camino que emplea la placenta humana para mantener la esteroidogénesis (van der Kant, Zondervan, Janssen \& Neefjes, 2013). En el laboratorio, hemos determinado que la proteína HSP60 comparte epítopes con la STARD3 y que su presencia induce el incremento en la síntesis de progesterona de las mitocondrias del sinciciotrofoblasto (Olvera-Sánchez, Espinosa-García, Monreal, Flores-Herrera \& Martínez, 2011). Recientemente se determinó que la HSP60 participa junto con la MLN64 en el transporte de colesterol para llevar a cabo la esteroidogénesis en la placenta humana (Monreal-Flores, Espinosa-García, García-Regalado, Arechavaleta-Velasco \& Martínez, 2017).

Se han descrito complejos multiproteicos responsables de conducir el colesterol hasta el P450scc, entre los que se encuentra el transduceosoma, compuesto de diversas proteínas membranales y citoplasmáticas y el metabolón esteroidogénico descrito como un conjunto de proteínas de las membranas mitocondriales (Fan \& Papadopoulos, 2013). Al respecto, se reportó que la STARD3 es capaz de generar puntos de contacto entre el RE y los endosomas, como un paso previo al transporte de colesterol a las mitocondrias (Alpy et al., 2013) y un mecanismo particular para la esteroidogénesis de la placenta humana (Tuckey, Bose, Czerwionka \& Miller, 2004; Martin, Kennedy \& Karten, 2014).

En su conjunto, la información muestra que de manera general el transporte de colesterol para la esteroidogénesis requiere de múltiples sistemas que involucran proteínas como la STARD1 y la STARD3, los endosomas tardíos, las gotas lipídicas, el citoesqueleto y los elementos MAMs, MINOS (mitocondrial inner rmembrane organizing system) y MCSs (mitocondrial contact sites), así como los sitios de contacto entre las membranas mitocondriales para realizar con éxito el flujo de colesterol y su transformación en pregnenolona en los tejidos esteroidogénicos de regulación aguda y en progesterona en el caso de la placenta humana, un tejido de regulación crónica.

\section{Sitios DE CONTACTO ENTRE LAS MEMBRANAS MITOCONDRIALES}

Hackenbrock \& Miller (1975), describieron por primera vez que las mitocondrias poseen diferentes tipos de sitios de contacto y que son complejos multienzimáticos que realizan diferentes funciones mitocondriales. Su formación se lleva a cabo mediante un proceso dinámico que requiere de la asociación de proteínas y enzimas específicas.

Para la fosforilación oxidativa se ha descrito la asociación de varias proteínas, entre las cuales están el acarreador de los adenín-nucleótidos (ANT), la porina o el canal aniónico dependiente del voltaje (VDAC), la creatina cinasa y la hexocinasa, entre otros (Adams, Bosch, Schelege, Wallimann \& Brdiczka, 1989; Papadopoulos, 1993). En la apoptosis, se asocian proteínas como el ANT, la VDAC, la ciclofilina D y la hexocinasa II. Además, la membrana externa posee proteínas que pueden establecer, junto con la actina, uniones dependientes de ATP y proteínas antiapoptóticas de la familia Bcl-2, cuya sobreexpresión tiene como consecuencia el bloqueo de la apoptosis (Adams \& Cory, 2001; Suen, Norris $\&$ Youle, 2008). Otro ejemplo es el poro de la transición de la permeabilidad (Rasola \& Bernardi, 2014), que es un complejo multiproteico que forma poros no selectivos en la membrana interna y que tiene los siguientes componentes estructurales: el ANT, la ciclofilina D, y el VDAC. Adicionalmente, se ha identificado al translocador de proteínas (TPSO, antes conocido como el receptor periférico a benzodiacepinas) unido al poro en la membrana externa, la creatina cinasa en el espacio intermembranal y la hexocinasa II ligadas a la VDAC en la cara citosólica de la membrana externa, así como las proteínas Bax/Bcl-2 (Zamzami \& Kroemer, 2001).

Finalmente se ha sugerido que para la esteroidogénesis en las glándulas suprarrenales y gónadas existe un punto de contacto específico, compuesto de un octámero de la creatina cinasa (CK), el VDAC, la TPSO y el ANT, además del IP3R (ERresident inositol triphosphate receptor), la Mfn2 y la Mfn1 (mitofusina 1 y 2), entre otras (Martin, Kennedy \& Karten, 2014). La asociación de las proteínas de la membrana externa como VDAC1 y TSPO con las proteínas de la familia de las ATPasas (ATAD3a) en la membrana interna, forman el núcleo de un complejo que regula la importación del colesterol mitocondrial (Issop et al., 2015). Datos recientes sugieren que la TPSO, aunque forma parte del sitio de contacto, no es indispensable para el transporte del colesterol (Tu et al., 2014; Morohaku et al., 2014). Adicionalmente, se ha descrito que la asociación entre las proteínas Tom22, Tim23, Tim 50, y la $3 \beta H S D 2$ puede repercutir en la esteroidogénesis (Pawlak, Prasad, Thomas, Whittal \& Bose, 2011; Rajapaksha et al., 2016).

Nuestro grupo de trabajo sugirió un modelo para la participación de la STARD3 en las mitocondrias de la placenta humana (Esparza-Perusquía et al., 2015) donde se propone que las gotas lipídicas contienen proteínas constituyentes de los complejos de fusión de membranas denominados SNARE (soluble N-ethylmaleimide-sensitive factor attachmentprotein receptor), entre las que incluyen las $\alpha$-SNAP, syntaxina 3, 7 y 12, syntaxina-Binding Protein-2, syntaxina-Binding Protein-3, y VAMP-8, que podrían estar participando en el transporte del colesterol en las mitocondrias de la placenta humana. Se ha demostrado que la proteína SNAP promueve la interacción entre las gotas de lípidos y las mitocondrias, y que en las células esteroidogénicas las SNARE se expresan como Syntaxina-17, 
SNAP-23 y SNAP-25 (Grant et al., 1999; Steegmaier, Oorschot, Klumperman \& Scheller, 2000; Jägerström et al., 2009). Estas observaciones sugieren que las proteínas SNARE podrían contribuir en el mecanismo de transporte del colesterol a las mitocondrias esteroidogénicas, muy probablemente mediante la promoción de la interacción funcional entre las gotas de lípidos, el retículo endoplásmico, los endosomas y las mitocondrias. En las células del sinciciotrofoblasto, la proteína STARD3 puede ser incorporada en las mitocondrias a través de estos complejos y ser transformada a su forma activa por proteasas mitocondriales (Esparza-Perusquía et al., 2015), favoreciendo su incorporación o participación en los sitios de contacto (Uribe, Strauss \& Martínez, 2003; Miller \& Bose 2011) y promover el transporte del colesterol para la síntesis de la progesterona (Strauss, Kishida, Christenson, Fujimoto \& Hiroi, 2003).

\section{AgRadecimientos}

Parte de este trabajo fue apoyado por el programa PAPIIT de la UNAM con los proyectos IN211912, IN211715, IN215518 y IN222617 y del proyecto 168025 de CONACYT.

\section{REFERENCIAS}

Adams, J.M. \& Cory, S. (2001). Life-or-death decisions by the Bcl-2 protein family. Trends Biochem. Sci., 26, 61-66. DOI: 10.1016/S0968-0004(00)01740-0.

Adams, V., Bosch, W., Schelege. J., Wallimann, T. \& Brdiczka, D. (1989). Further characterization of contact sites from mitochondria of different tissues: topology of peripheral kinases. Biochim. Biophys. Acta, 981(2), 213-225. DOI: 10.1016/0005-2736(89)90031-X.

Alpy, F. \& Tomasetto, C. (2014). START ships lipids across interorganelle space. Biochimie, 96, 85-95. DOI: 10.1016/j.biochi.2013.09.015.

Alpy, F., Rousseau, A., Schwab, Y., Legueux, F., Stoll, I., Wendling, C., Spiegelhalter, C., Kessler, P., Mathelin, C., Rio, M.C., Levine, T.P. \& Tomasetto, C. (2013). STARD3 or STARD3NL and VAP form a novel molecular tether between late endosomes and the ER. J. Cell Sci., 126, 5500-5512. DOI: 10.1242/jcs. 139295.

Ashary, N., Tiwari, A. \& Modi, D. (2018). Embryo Implantation: Warin times of love. Endocrinology, 159, 1188-1198. DOI: 10.1210/en.2017-03082.

Baardman, M.E., Kerstjens-Frederikse, W.S., Berger, R.M.F., Bakker, M.K., Hofstra, R.M.W. \& Plösch, T. (2013).The role of maternal-fetal cholesterol transport in early fetal life: current insights. Biol. Reprod., 88(24), 1-9. DOI: 10.1095/biolreprod.112.102442.

Bartels, A. \& O’Donoghue, K. (2011). Cholesterol in pregnancy: a review of knowns and unknowns. Obstet. Med., 4, 147-151. DOI: 10.1258/om.2011.110003.

Beller, M., Thiel, K., Thul, P.J. \& Jäckle, H. (2010). Lipid droplets: A dynamic organelle moves into focus. FEBS Lett, 584, 2176-2182. DOI: 10.1016/j.febslet.2010.03.022.
Bhattacharjee, J., Ietta, F., Romagnoli, R., Bechi, N., Caniggia, I. \& Paulesu, L. (2012). ABC transporters in human placenta and their role in maternal-fetal cholesterol transfer: ABCA1 candidate target. En Jing Zheng (Ed.). Recent Advances in Research on the Human Placenta (pp. 336-354).London: IntechOpen. ISBN 978-953-510194-9 DOI: $10.5772 / 1211$.

Bianco-Miotto, T., Blundell, C., Buckberry, S., Chamley, L., Chong, S., Cottrell, E., Dawson, P., Hanna, C., Holland, O., Lewis, R.M., Moritz, K., Myatt, L., Perkins, A.V., Powell, T., Saffery, R., Sferruzzi-Perri, A., Sibley, C., Simmons, D. \& O'Tierney-Ginn, P.F. (2016). IFPA meeting 2015 workshop report I: Placental mitochondrial function, transport systems and epigenetics. Placenta, 48, Suppl. 1, Trophoblast Res., 30, S3-S6. DOI: 10.1016/j. placenta.2015.11.014.

Bose, M., Whittal, R.M., Miller, W.L. \& Bose, H.S. (2008). Steroidogenic activity of StAR requires contact with mitochondrial VDAC1 and phosphate carrier protein. J. Biol. Chem., 283, 8837-8845. DOI: 10.1074/jbc. M709221200.

Burton, G.J. \& Fowden, A.L. (2015). The placenta: a multifaceted, transient organ. Phil. Trans. R. Soc. B, 370: 20140066. DOI: 10.1098/rstb.2014.0066.

Byrns, M.C. (2014). Regulation of progesterone signaling during pregnancy: Implications for the use of progestins for the prevention of preterm birth. J. Steroid Biochem.\& Mol. Biol., 139, 173- 181. DOI: 10.1016/j. jsbmb.2013.01.015.

Chatuphonprasert, W., Jarukamjorn, K. \& Ellinger, I. (2018). Physiology and pathophysiology of steroid biosynthesis, transport and metabolism in the human placenta. Front. Pharmacol., 9, 1027. DOI: 10.3389/fphar.2018.01027.

Chien, Y., Rosal, K. \& Chung, B. (2017). Function of CYP11A1 in the mitochondria. Mol. Cell Endocrinol., 441, 55-61. DOI: 10.1016/j.mce.2016.10.030.

Esparza-Perusquía, M., Olvera-Sánchez, S., Flores-Herrera, O., Flores-Herrera, H., Guevara-Flores, A., Pardo, J.P., Espinosa-García, M.T. \& Martínez, F. (2015). Mitochondrial proteases act on STARD3 to activate progesterone synthesis in human syncytiotrophoblast. Biochim. Biophys. Acta, 1850(1), 107-117. DOI: 10.1016/j.bbagen.2014.10.009.

Fan, J. \& Papadopoulos, V. (2013). Evolutionary origin of the mitochondrial cholesterol transport machinery reveals a universal mechanism of steroid hormone biosynthesis in animals. PLoS ONE, 8, e76701. DOI:10.1371/journal. pone.0076701.

Flis, V.V. \& Daum, G. (2013). Lipid transport between the endoplasmic reticulum and mitochondria. Cold Spring Harb Perspect Biol., 5(6), pii: a013235. DOI: 10.1101/ cshperspect.a013235.

Furuhashi, M., Seo, H., Mizutani, S., Narita, O., Tomoda, Y. \&, Matsui, N. (1989). Expression of low density lipoprotein 
receptor gene in human placenta during pregnancy. Mol. Endocrinol., 3, 1252-1256. DOI: 10.1210/mend-3-8-1252.

Galluzzi, L., Kepp, O. \& Kroemer, G. (2012). Mitochondria: master regulators of danger signalling. Nat. Rev. Moll. Cell Biol., 13, 780-788. DOI: 10.1038/nrm3479. *

Grant, N.J., Hepp, R., Krause, W., Aunis, D., Oehme, P. \& Langley, K. (1999). Differential expression of SNAP25 isoforms and SNAP-23 in the adrenal gland. $J$. Neurochem., 72(1), 363-372. DOI: 10.1046/j.14714159.1999.0720363.x.

Hackenbrock, C.R. \& Miller, K.J. (1975). The distribution of anionic sites on the surfaces of mitochondrial membranes. Visual probing with polycationic ferritin. J. Cell. Biol., 65(3), 615-630. DOI: 10.1083/jcb.65.3.615.

Halasz, M. \& Szekeres-Bartho, J. (2013). The role of progesterone in implantation and trophoblast invasion. J. Reprod. Immunol., 97, 43-50. DOI: 10.1016/j. jri.2012.10.011.

Holland, O., Nitert, M.D., Gallo, L.A., Vejzovic, M., Fisher, J.J. \& Perkins A.V. (2017a). Placental mitochondrial function and structure in gestational Disorders. Placenta, 54, 2-9.DOI: 10.1016/j.placenta.2016.12.012.

Holland, O.J., Hickey, A.J.J., Alvsaker, A., Moran, S., Hedges, C., Chamley, L.W. \& Perkins, A.V. (2017b). Changes in mitochondrial respiration in the human placenta over gestation. Placenta, 57, 102-112. DOI: 10.1016/j. placenta.2017.06.011.

Horvath, S.E. \& Daum, G. (2013). Lipids of mitochondria. Prog. Lipid Res., 52, 590-614. DOI: 10.1016/j. plipres.2013.07.002.

Issop, L., Fan, J., Lee, S., Rone, M.B., Basu, K., Mui, J. \& Papadopoulos, V. (2015).Mitochondria-associated membrane formation in hormone-stimulated Leydig cell steroidogenesis: Role of ATAD3. Endocrinology, 1: 334345. DOI: 10.1210/en.2014-1503.

Jägerström, M.S., Polesie, S., Wickström, Y., Johansson, B.R., Schroder, H.D., Højlund, K. \& Boström, P. (2009). Lipid droplets interact with mitochondria using SNAP23. Cell Biol. Int., 33(9), 934-940. DOI: 10.1016/j. cellbi.2009.06.011.

Kaiser, J. (2014). Gearing up for a closer look at the human placenta. Science, 344(6188), 1073. DOI: 10.1126/ science.344.6188.1073.

LaVoie, H.A. \& King, S.R. (2009). Transcriptional regulation of steroidogenic genes: STARD1, CYP11A1 and HSD3B. Exp. Biol. Med. (Maywood), 234, 880-907. DOI: 10.3181/0903-MR-97.

Marriott, K.S., Prasad, M., Thapliyal, V. \& Bose, H.S. (2012). $\sigma-1$ Receptor at the mitochondrial-associated endoplasmic reticulum membrane is responsible for mitochondrial metabolic regulation. J. Pharmacol. Exp.Ther., 343, 578586. DOI: $10.1124 /$ jpet.112.198168.

Martin, L.A., Kennedy, B.E. \& Karten, B. (2014). Mitochondrial cholesterol: mechanisms of import and effects on mitochondrial function. J. Bioenerg. Biomembr., 48(2), 137-151. DOI: 10.1007/s10863-014-9592-6.

Martínez, F. \& Strauss, J.F. 3rd (1997). Regulation of mitochondrial cholesterol metabolism. Subcell. Biochem., 28, 205-234. PMID: 9090296.

Mesmin, B., Antonny, B. \& Drin, G. (2013). Insights into the mechanisms of sterol transport between organelles. Cell. Mol. Life Sci., 70, 3405-3421.DOI: 10.1007/s00018-0121247-3.

Miller, W.L. \& Bose, H.S. (2011). Early steps in steroidogenesis: intracellular cholesterol trafficking. $J$. Lipid Res., 52, 2111-2135. DOI: 10.1194/jlr.R016675.

Miller, W.L. (2013). Steroid hormone synthesis in mitochondria. Mol. Cell Endocrinol., 379, 62-73. DOI. 10.1016/j.mce.2013.04.014.

Monreal-Flores, J., Espinosa-García, M.T., García-Regalado, A., Arechavaleta-Velasco, F. \& Martínez, F. (2017). The heat shock protein 60 promotes progesterone synthesis in mitochondria of JEG-3 cells. Reprod. Biol., 17, 154-161. DOI: 10.1016/j.repbio.2017.04.001.

Morel, Y., Roucher, F., Plotton, I., Goursaud, C., Tardy, V. \& Mallet, D. (2016). Evolution of steroids during pregnancy: Maternal, placental and fetal synthesis. Ann. Endocrinol. (Paris), 77(2), 82-89. DOI: 10.1016/j.ando.2016.04.023. 2016.

Morohaku, K., Pelton, S.H., Daugherty, D.J., Butler, W.R., Deng, W. \& Selvaraj, V. (2014). Translocator protein/ peripheral benzodiazepine receptor is not required for steroid hormone biosynthesis. Endocrinology, 155(1), 89-97. DOI: 10.1210/en.2013-1556.

Navarrete, J., Flores-Herrera, O., Uribe, A. \& Martínez, F. (1999). Differences in cholesterol incorporation into mitochondria from hepatoma AS-30D and human term placenta. Placenta, 20(4), 285-291. DOI: 10.1053/ plac.1998.0374.

Olvera-Sánchez, S., Espinosa-García, M.T., Monreal, J., Flores-Herrera, O. \& Martinez, F. (2011). Mitochondrial heat shock protein participates in placental steroidogenesis. Placenta, 32, 222-229. DOI: 10.1016/j. placenta.2010.12.018.

Pagler, T.A., Golsabahi, S., Doringer, M., Rhode, S., Schutz, G.J., Pavelka, M., Wadsack, C., Gauster, M., Lohninger, A., Laggner, H., Strobl, W. \& Stangl, H. (2006). A Chinese hamster ovarian cell line imports cholesterol by high density lipoprotein degradation. J. Biol. Chem., 281, 38159-38171. DOI: 10.1074/jbc.M603334200

Papadopoulos, V. \& Miller, W.L. (2012). Role of mitochondria in steroidogenesis. Best Pract. Res. Clin. Endocrinol. Metab., 26(6), 771-790. DOI: 10.1016/j. beem.2012.05.002.

Papadopoulos, V. (1993). Peripheral-type benzodiazepine/ diazepam binding inhibitor receptor: biological role in steroidogenic cell function. Endocr. Rev., 14, 222-240. DOI: 10.1210 /edrv-14-2-222. 
Paul, S., Gupta, P.D., Jailkhani, B.L. \& Talwar, G.P. (1980). Resistance of human syncytiotrophoblast to hypotonic and thermal stress. J. Reprod. Fertil., 58(1), 183-187. PMID: 7359476.

Paul, S., Jailkhani, B.L. \& Talwar, G.P. (1978). Isolation and functional maintenance in culture of syncytiotrophoblasts from human placenta. Indian J. Exp. Biol., 16, 12261235. PMID: 750412.

Pawlak, K.J., Prasad, M., Thomas, J.L., Whittal, R.M. \& Bose, H.S. (2011). Inner mitochondrial translocase Tim50 interacts with $3 \beta$-hydroxysteroid dehydrogenase type 2 to regulate adrenal and gonadal steroidogenesis. J. Biol.Chem., 286(45), 39130-39140. DOI: 10.1074/jbc. M111.290031.

Poderoso, C., Duarte, A., Cooke, M., Orlando, U., Gottifredi, V., Solano, A.R. Lemos, J.R. \& Podestá, E.J. (2013). The spatial and temporal regulation of the hormonal signal. Role of mitochondria in the formation of a protein complex required for the activation of cholesterol transport and steroids synthesis. Mol. Cell. Endocrinol., 371, 26-33. DOI: 10.1016/j.mce.2012.12.024.

Prasad, M., Kaur, J., Pawlak, K.J., Bose, M., Whittal, R.M. \& Bose, H.S. (2015). Mitochondria associated ERMembrane (MAM) regulates steroidogenic activity via StAR-VDAC2 interaction. J. Biol. Chem., 290, 26042616. DOI: $10.1074 / j b c . M 114.605808$.

Rajapaksha, M., Kaur, J., Bose, M., Whittal, R.M. \& Bose, H.S. (2013). Cholesterol-mediated conformational changes in the steroidogenic acute regulatory protein are essential for steroidogenesis. Biochemistry, 52, 7242-7253. DOI: $10.1021 /$ bi401125v.

Rajapaksha, M., Kaur, J., Prasad, M., Pawlak, K.J., Marshall, B., Perry, E.W., Whittal, R.M. \& Bose, H.S. (2016). An outer mitochondrial translocase, Tom22, is crucial for inner mitochondrial steroidogenic regulation in adrenal and gonadal tissues. Mol. Cell Biol., 36(6), 1032-1047. DOI: 10.1128/MCB.01107-15;

Rasola, A. \& Bernardi, P. (2014). The mitochondrial permeability transition pore and its adaptive responses in tumor cells. Cell Calcium, 56(6), 437-445. DOI: 10.1016/j.ceca.2014.10.003.

Sanderson, J.T. (2009). Placental and Fetal Steroidogenesis Human. En: Lafond, J. \& Vaillancourt, C. (Ed.). Embryogenesis: Methods and Protocols. (pp. 127-136) Nueva York:HumanaPress.DOI 10.1007/978-1-60327009-0 7.

Simpson, E.R. \& MacDonald, P.C. (1981). Endocrine physiology of the placenta. Annu. Rev. Physiol., 43, 163188. DOI: 10.1146/annurev.ph.43.030181.001115

Soffientini, U. \& Graham, A. (2016). Intracellular cholesterol transport proteins: roles in health and disease. Clin. Sci., 130, 1843-1859 DOI: $10.1042 / C S 20160339$

Steegmaier, M., Oorschot, V., Klumperman, J. \& Scheller, R.H. (2000). Syntaxin 17 is abundant in steroidogenic cells and implicated in smooth endoplasmic reticulum membrane dynamics. Mol. Biol. Cell, 11(8), 2719-2731. DOI: $10.1091 / \mathrm{mbc} .11 .8 .2719$.

Strauss, J.F.3rd, Kishida, T., Christenson, L.K., Fujimoto, T. \& Hiroi, H. (2003). StAR domain proteins and the intracellular trafficking of cholesterol in steroidogenic cells. Mol. Cell, Endocrinol., 202, 59-65. DOI: 10.1016/ S0303-7207(03)00063-7.

Suen, D.F., Norris, K.L. \& Youle, R.J. (2008). Mitochondrial dynamics and apoptosis. Genes Dev., 22, 1577-1590. DOI: $10.1101 /$ gad.1658508.

Tait, S.W. \& Green, D.R. (2012). Mitochondria and cell signalling. J. Cell Sci., 125, 807-815. DOI: 10.1242/ jes.099234.

Tu, L.N., Morohaku, K., Manna, P.R., Pelton, S.H., Butler, W.R., Stocco, D.M. \& Selvaraj, V. (2014). Peripheral benzodiazepine receptor/translocator protein global knock-out mice are viable with no effects on steroid hormone biosynthesis. J. Biol. Chem., 289, 40, 27444 27454. DOI: $10.1074 / j b c . M 114.578286$.

Tuckey, R.C., Headlam, M.J, Bose, H.S. \& Miller, W.L. (2002). Transfer of cholesterol between phospholipid vesicles mediated by the steroidogenic acute regulatory protein (StAR). J. Biol. Chem., 277, 47123-28. DOI: 10.1074/jbc.M206965200.

Tuckey, R.C., Bose, H.S., Czerwionka, I. \& Miller, W.L. (2004). Molten globule structure and steroidogenic activity of N-218 MLN64 in human placental mitochondria. Endocrinology, 145, 1700-1707. DOI: 10.1210/en.20031034.

Uribe, A. Strauss, J.F. 3rd \& Martínez, F. (2003). Contact sites from human placental mitochondria: characterization and role in progesterone synthesis. Arch. Biochem. Biophys., 413(2), 172-181. DOI: 10.1016/S00039861(03)00097-3.

van der Kant, R., Zondervan, I., Janssen, L. \& Neefjes, J. (2013). Cholesterol-binding molecules MLN64 and ORP1L mark distinct late endosomes with transporters ABCA3 and NPC1. J. Lipid Res., 54, 2153-2165. DOI: 10.1194/jlr.M037325

van Leusden, H. \& Villee, C.A. (1965).The de novo synthesis of sterols and steroids -from acetate by preparations of human term placenta. Steroids, 6, 31-45. DOI: 10.1016/0039-128X(65)90031-0.

Vaughan, O.R. \& Fowden, A.L. (2016). Placental metabolism: substrate requirements and the response to stress. Reprod. Dom. Anim., 51 (Suppl. 2), 25-35.DOI: 10.1111/ rda.12797.

Wadsack, C., Hammer, A., Levak-Frank, S., Desoye, G., Kozarsky, K.F., Hirschmugl, B., Sattler, W. \& Malle, E. (2003). Selective cholesteryl ester uptake from high density lipoprotein by human first trimester and term villous trophoblast cells. Placenta, 24, 31-43. DOI: 10.1053/plac.2002.0912. 
Watari, H., Arakane, F., Moog-Lutz, C., Kallen, C.B., Tomasetto, C., Gerton, G.L., Rio, M.C., Baker, M.E. \& Strauss, J.F. 3rd. (1997). MLN64 contains a domain with homology to the steroidogenic acute regulatory protein (StAR) that stimulates steroidogenesis. Proc. Natl. Acad. Sci. USA, 94(16), 8462-8467doi.org/10.1073/ pnas.94.16.8462

Winkel, C.A., MacDonald, P.C. \& Simpson, E.R. (1981). The role of receptor-mediated low-density lipoprotein uptake and degradation in the regulation of progesterone biosynthesis and cholesterol metabolism by human trophoblasts. Placenta, (Suppl. 3), 133-143. PMID: 6306642.

Winkel, C.A., Snyder, J.M., MacDonald, P.C. \& Simpson, E.R. (1980). Regulation of cholesterol and progesterone synthesis in human placental cells in culture by serum lipoproteins. Endocrinology, 106, 1054-1060. DOI: 10.1210/endo-106-4-1054.

Woollett, L.A. (2011). Transport of maternal cholesterol to the fetal circulation. Placenta, 32 (Suppl. 2), S218-21. DOI: 10.1016/j.placenta.2011.01.011.

Yang, H., Galea, A., Sytnyk, V. \& Crossley, M. (2012). Controlling the size of lipid droplets: lipid and protein factors. Curr. Opin. Cell Biol., 24, 509-516. DOI: 10.1016/j.ceb.2012.05.012.

Zelewski, L. \& Villee, C.A. (1966). The biosynthesis of squalene, lanosterol, and cholesterol by minced human placenta. Biochemistry, 5,1805-1814. PMID: 5963423.

Zamzami, N. \& Kroemer, G. (2001). The mitochondrion in apoptosis: how Pandora's Box opens. Nat. Rev. Mol. Cell Biol., 2(1), 67-71.DOI: 10.1038/35048073 